\title{
Stabilizing unstable systems by the act-and-wait concept - Case studies
}

\author{
Tamás Insperger Gábor Stépán \\ Budapest University of Technology and Economics, Department of \\ Applied Mechanics, Budapest, Hungary \\ (e-mail: inspi@mm.bme.hu, stepan@mm.bme.hu)
}

\begin{abstract}
The act-and-wait control is a special time-periodic control for systems with feedback delay, where the control gains are periodically switched on and off in order to neutralize the destabilizing effect of the delay. In this paper, we present two case studies to show how the actand-wait type time-periodic control can be used to stabilize otherwise unstable systems. The first case study is a second order system subjected a PD controller with large feedback delay such that the system is always unstable if the feedback is continuously applied with constant gains. The second case study is a first order system subjected to the feedback of the second derivative of the state resulting in a differential equation of advanced type. It is shown that both unstable systems can be stabilized using the act-and-wait control concept.
\end{abstract}

Keywords: feedback delay, periodic control, stabilization, pole assignment, stability domains

\section{INTRODUCTION}

Consider the linear system

$$
\begin{aligned}
\dot{x}(t) & =A x(t)+B u(t), \\
y(t) & =C x(t)
\end{aligned}
$$

with $x(t) \in \mathbb{R}^{n}, u(t) \in \mathbb{R}^{m}, y(t) \in \mathbb{R}^{l}$. Consider the delayed feedback controller

$$
u(t)=D y(t-\tau)
$$

where $\tau$ is the time delay in the feedback loop. We assume that the delay is a fixed parameter of the control system and cannot be eliminated or tuned during the control design. There are several sources of such time delays, e.g., acquisition of response and excitation data, information transmission, on-line data processing, computation and application of control forces.

System (1)-(2) with controller (3) implies the delay differential equation (DDE)

$$
\dot{x}(t)=A x(t)+B D C x(t-\tau) .
$$

Due to the time delay, system (4) has infinitely many poles (called also characteristic roots or characteristic exponents) determined by the transcendental characteristic equation

$$
\operatorname{det}\left(\lambda I-A-B D C \mathrm{e}^{-\tau \lambda}\right)=0 .
$$

The system is asymptotically stable if all the poles are located in the left half of the complex plane. Stability conditions for the system's parameters can be given by monitoring the number of unstable poles (see, e.g., Stépán, 1989, Atay, 1999, Olgac and Sipahi, 2002, Michiels and Roose, 2003). The difficulty of this problem is that infinitely many poles should be controlled by finite number of control parameters, i.e., by the elements of matrix $D$.

\footnotetext{
* This work was partially supported by the János Bolyai Research Scholarship of the Hungarian Academy of Sciences and the Hungarian National Science Foundation under grant no. T068910 and K72911.
}

An effective way of managing pole placement problem is the use of time-varying controllers. The problem of stabilization by means of time-varying feedback gains in non-delayed systems has been presented by Brockett (1998) as one of the challenging open problems in control theory. For non-delayed systems (i.e., for $\tau=0$ ), the Brockett problem can be posed as:

Problem 1. For given matrices $A, B$ and $C$, under what circumstances does there exist a time-varying controller

$$
u(t)=G(t) y(t),
$$

such that the system is asymptotically stable?

For systems with no feedback delay, results have been presented by Leonov (2002) and by Allright et al. (2005) for piecewise constant control gains and by Moreau and Aeyels (2004) for sinusoidal control gains. A comparison of delayed feedback and time-varying gains was provided by Michiels et al. (2004). The solution to the problem for a wide class of systems - without delay - was presented by Boikov (2005).

For systems with feedback delay, the Brockett problem can be composed as:

Problem 2. For given matrices $A, B$ and $C$ and for given feedback delay $\tau$, under what circumstances does there exist a time-varying controller

$$
u(t)=G(t) y(t-\tau),
$$

such that the system is asymptotically stable?

This stabilization problem is quite complicated since the system has infinitely many poles that all should be monitored during the stabilization process. One possible approach to the problem is the application of the act-andwait control technique introduced by Insperger (2006) for continuous-time systems and by Insperger and Stépán (2007) for discrete-time systems. 
In this paper, stabilization of unstable systems using the act-and-wait concept is demonstrated in case studies. The first case study is a second order system subjected to a PD controller with a large delay in the feedback loop. The second case study is a first order system subjected to the feedback of the second derivative of the state that, in fact, corresponds to a differential equation of advanced type.

\section{THE ACT-AND-WAIT CONTROL CONCEPT}

In this section, the act-and-wait control concept is briefly summarized according to Insperger (2006). Consider the time-varying controller (7) with the $T$-periodic act-andwait matrix

$$
G(t)=\left\{\begin{array}{ccc}
0 & \text { if } & 0 \leq t \bmod T<t_{w} \\
\Gamma(t) & \text { if } & t_{w} \leq t \bmod T<t_{w}+t_{a}=T
\end{array} .\right.
$$

Using time-varying controller (7) with the act-and-wait matrix (8) instead of the continuous controller (3), the delayed feedback term is switched off for period of length $t_{w}$ (wait), and it is switched on for period of length $t_{a}$ (act).

System (1) with controller (7) can be written in the form

$$
\dot{x}(t)=A x(t)+B G(t) C x(t-\tau),
$$

which is a time-periodic DDE. According to the Floquet theory of DDEs, stability is determined by the nonzero eigenvalues of the system's monodromy operator, called also Floquet multipliers or poles. The system is asymptotically stable if all the (usually) infinitely many Floquet multipliers are located inside the unit circle of the complex plane.

The merit of the act-and-wait concept lies in the fact that if $t_{w} \geq \tau$ in (8) then equation (9) can be solved step-bystep as an ODE, and the state at any instant $t>0$ can be given in the form $x(t)=\Phi(t) x(0)$. The solution matrix $\Phi(t)$ can be constructed as

$$
\Phi(t)=\left\{\begin{array}{ccc}
\mathrm{e}^{A t} & \text { if } & 0 \leq t<t_{w} \\
\Psi_{h}(t) & \text { if } & t_{w}+(h-1) \tau<t \leq t_{w}+h \tau
\end{array},\right.
$$

where $h=1,2, \ldots$ and $\Psi_{h}(t)$ can be given in the recursive form

$$
\begin{gathered}
\Psi_{1}(t)=\mathrm{e}^{A t}+\int_{t_{w}}^{t} \mathrm{e}^{A(t-s)} B \Gamma(s) C \mathrm{e}^{A(s-\tau)} \mathrm{d} s \\
\Psi_{2}(t)=\mathrm{e}^{A\left(t-t_{w}-\tau\right)} \Psi_{1}\left(t_{w}+\tau\right) \\
\quad+\int_{t_{w}+\tau}^{t} \mathrm{e}^{A(t-s)} B \Gamma(s) C \Psi_{1}(s-\tau) \mathrm{d} s \\
\vdots \\
\Psi_{h+1}(t)=\mathrm{e}^{A\left(t-t_{w}-h \tau\right)} \Psi_{h}\left(t_{w}+h \tau\right) \\
\quad+\int_{t_{w}+h \tau}^{t} \mathrm{e}^{A(t-s)} B \Gamma(s) C \Psi_{h}(s-\tau) \mathrm{d} s .
\end{gathered}
$$

$$
\text { If } \begin{array}{r}
t_{w}+(h-1) \tau<T \leq t_{w}+h \tau \text { then } \\
\Phi(T)=\Psi_{h}(T) .
\end{array}
$$

Thus, the monodromy operator of the system is represented by the $n \times n$ matrix $\Phi(T)$, and the stability of the system is determined by the $n$ eigenvalues of $\Phi(T)$. This way, the infinite dimensional pole placement problem is reduced to a finite dimensional one: $n$ eigenvalues of
$\Phi(T)$ should be placed inside the unit circle of the complex plane.

\section{STABILIZATION OF A SECOND ORDER SYSTEM}

Consider the second-order system subjected to a PD controller with a unit delay $\tau=1$ in the feedback loop

$$
\ddot{\varphi}(t)+a \varphi(t)=-p \varphi(t-1)-d \dot{\varphi}(t-1) .
$$

This system corresponds to the system described by (1)(2) and by the time-invariant controller (3) with

$$
\begin{gathered}
A=\left[\begin{array}{cc}
0 & 1 \\
-a & 0
\end{array}\right], \quad B=\left[\begin{array}{l}
0 \\
1
\end{array}\right], \quad C=\left[\begin{array}{ll}
1 & 0 \\
0 & 1
\end{array}\right], \\
D=\left[\begin{array}{l}
-p \\
-d
\end{array}\right]^{T}, \quad \tau=1 .
\end{gathered}
$$

The corresponding characteristic equation reads

$$
\lambda^{2}+a+p \mathrm{e}^{-\lambda}+d \lambda \mathrm{e}^{-\lambda}=0
$$

This transcendental equation has infinitely many poles that cannot arbitrarily be placed using the control gains $p$ and $d$. Moreover, if $a<-2$ then the system cannot even be stabilized, it is unstable for all $(p, d)$ pairs (see, e.g., Stépán, 1989). Here, we consider the case $a=-3$, that is, the system cannot be stabilized using the time-invariant controller (3). However, it can be stabilized using the actand-wait controller as it is shown below.

Consider now the same system subjected to the act-andwait controller in the form

$$
\ddot{\varphi}(t)+a \varphi(t)=g(t)(-p \varphi(t-1)-d \dot{\varphi}(t-1)),
$$

where

$$
g(t)=\left\{\begin{array}{llc}
0 & \text { if } & 0 \leq t \bmod T<t_{w} \\
1 & \text { if } & t_{w} \leq t \bmod T<t_{w}+t_{a}=T
\end{array}\right.
$$

is the act-and-wait switching function. Here, the feedback is zero for the waiting period, and constant proportional and differential gains are applied in the acting period. This system corresponds to the system described by (1)-(2) and by the time-varying controller (7) with (8) such that $\Gamma(t) \equiv D$. If $t_{w} \geq \tau$, then the system can be described by a $2 \times 2$ monodromy matrix $\Phi(T)$ that can be obtained according to (11) (note that $n=2$ ). In this case only two poles, the two eigenvalues of $\Phi(T)$, should be placed inside the unit circle by tuning the control gains $p$ and $d$ and the lengths $t_{a}$ and $t_{w}$ of the acting and waiting periods.

Figure 1 shows a series of stability charts for the system in the plane of $(p, d)$ for different values of $t_{a}$ and $t_{w}$. The charts were determined numerically by computing the critical (maximum in modulus) poles over a $200 \times 200$ sized grid of the parameters $p$ and $d$. For the cases $t_{w} \geq \tau$, the eigenvalues of the $2 \times 2$ monodromy matrix $\Phi(T)$ was computed numerically. If $t_{w}=0.8<\tau$ then the formula (10) is not valid, for this cases, the semi-discretization method was used to compute the stability boundaries.

Since the act-and-wait period $T=t_{w}+t_{a}$ is different for each chart, the decay ratio $\rho=\left|\mu_{1}\right|^{1 / T}$ is used to compare different cases, where $\mu_{1}$ is the critical Floquet multiplier. This decay ratio characterizes the decay over a unit time step

$$
\|x(t+1)\| \leq \rho\|x(t)\|
$$

In Fig. 1, the contour lines where the decay ratio are in modulus equal to $1,2,3, \ldots$ are also presented. The 
$t_{a}=0.2$
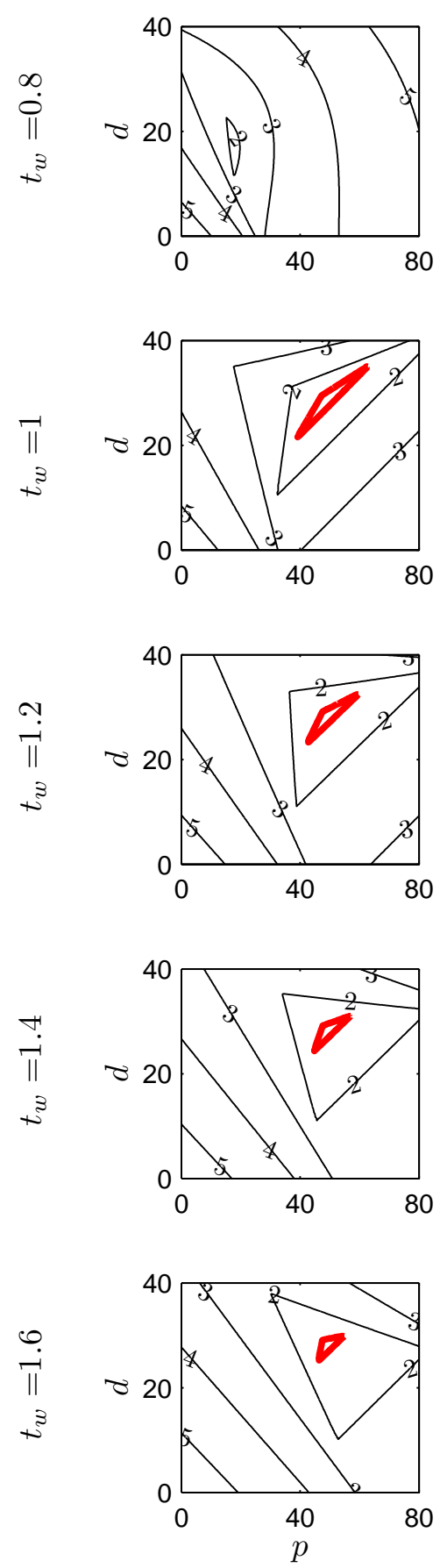

$t_{a}=0.4$
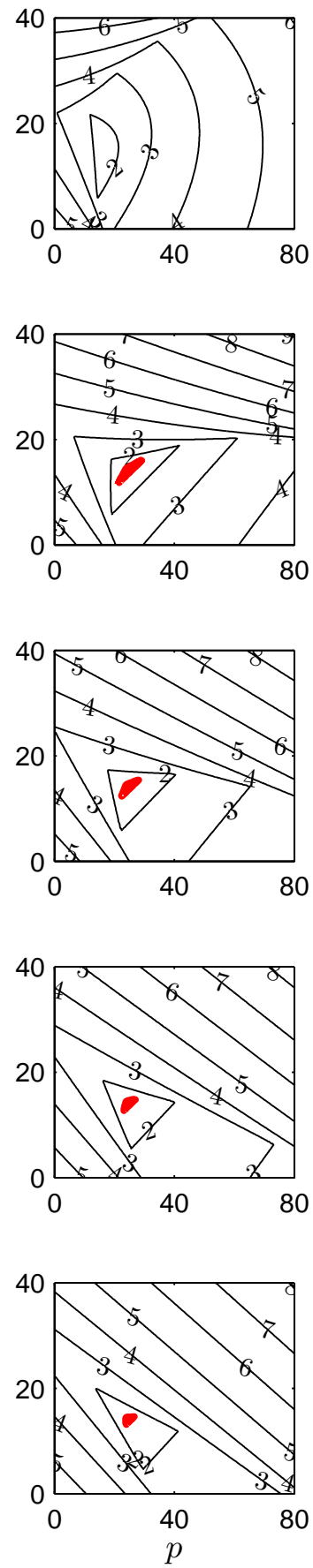

$t_{a}=0.6$
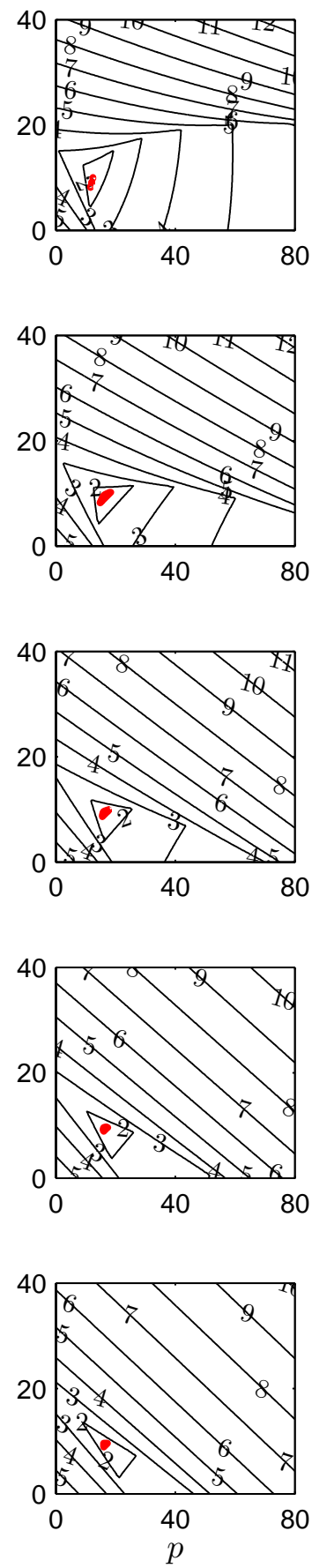

$t_{a}=0.8$
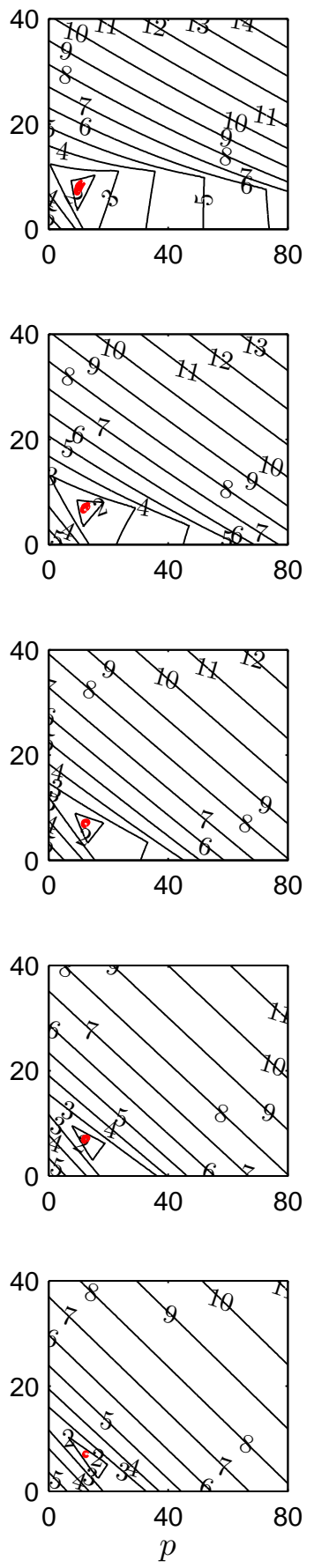

Fig. 1. Stability charts for system (16) with (17) for $a=-3$. Contour lines of the decay ratio are presented by thin black lines, stability boundaries are denoted by red thick lines.

stability boundaries, where the magnitude of the critical poles are just equal to 1 are denoted by thick red lines.

As is can be seen in Fig. 1, system (16) with (17) can be stabilized for different combinations of the control parameters $p, d, t_{w}$ and $t_{a}$. It can also be seen that some narrow stable domains occurs in the $(p, d)$ plane even if $t_{w}<\tau$. That is, the condition $t_{w} \geq \tau$ is not a necessary condition for the stability. The stable domains in the charts of Fig. 1 seems to be quite narrow, but note that system (12) with continuously active feedback is always unstable for any $(p, d)$ pair. 


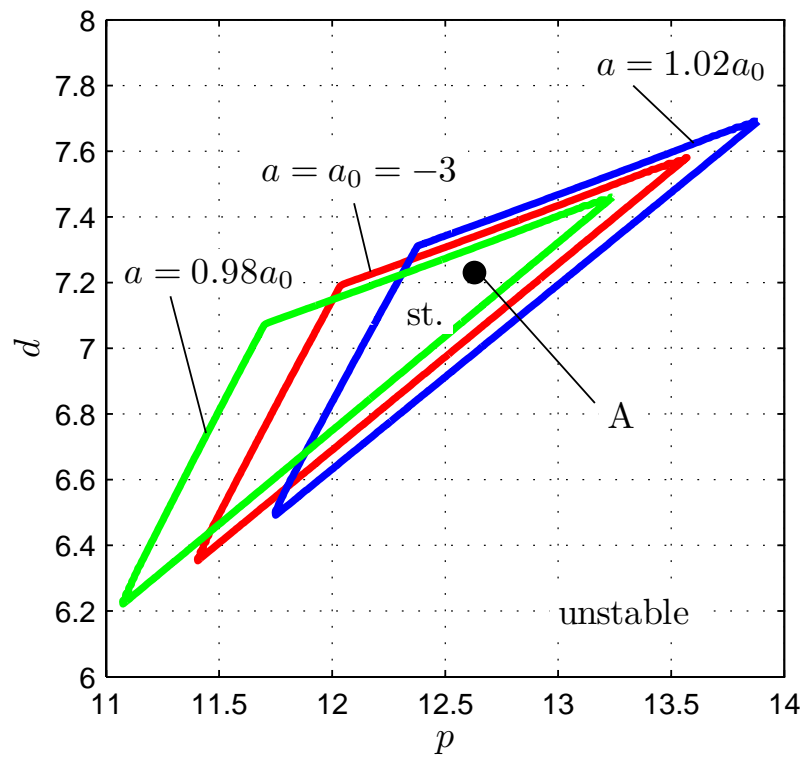

Fig. 2. Enlarged stability charts for system (16) with (17), $t_{w}=1, t_{a}=0.8, a$ is perturbed by \pm 2 and $4 \%$ around $a_{0}=-3$.

Figure 2 presents the enlarged stability diagrams for $t_{w}=$ 1 and $t_{a}=0.8$ in order to show the sensitivity of the control technique with respect to the system parameter $a$. The stability boundaries were determined for 2 and $4 \%$ perturbations of the system parameter $a$, i.e, for $a=$ $-2.94,-3.06$ and $-2.88,-3.12$. It can be seen that there exist such control gains $p$ and $d$ for that the system remains stable even for $4 \%$ perturbation of $a$. This point is denoted by A in Fig. 2. The corresponding time-domain simulations for different perturbations can be seen in Fig. 3. Thick lines denote the periods of acting and thin lines denote waiting. It can be seen that $\varphi(t)$ grows exponentially in the first waiting period $\left[0, t_{w}\right)$ since the open loop system is unstable, then, during the first acting period $\left[t_{w}, T\right)$, the growing tendency of $\varphi(t)$ is reversed.

\section{STABILIZATION OF A DIFFERENTIAL EQUATION OF ADVANCED TYPE}

Consider the scalar equation

$$
\dot{x}(t)=a x(t)+b u,
$$

assume that the only available output of the system is

and the feedback law is

$$
y(t)=c \ddot{x}(t),
$$

$$
u(t)=d y(t-1)
$$

with feedback delay being $\tau=1$. This system is different from the one described by (1)-(2) with (3), since here the output is equal to the second derivative of the state. Equations (19)-(21) can be put in the form

$$
\dot{x}(t)=a x(t)+d \ddot{x}(t-1),
$$

which is a differential equation of advanced type. (Here, without loss of generality, we assumed that $b=1$ and $c=1$.) For equations of advanced type, there is always at least one root of the characteristic equation with positive real part, therefore these equations are always unstable (see, e.g., Èl'sgol'c, 1964).
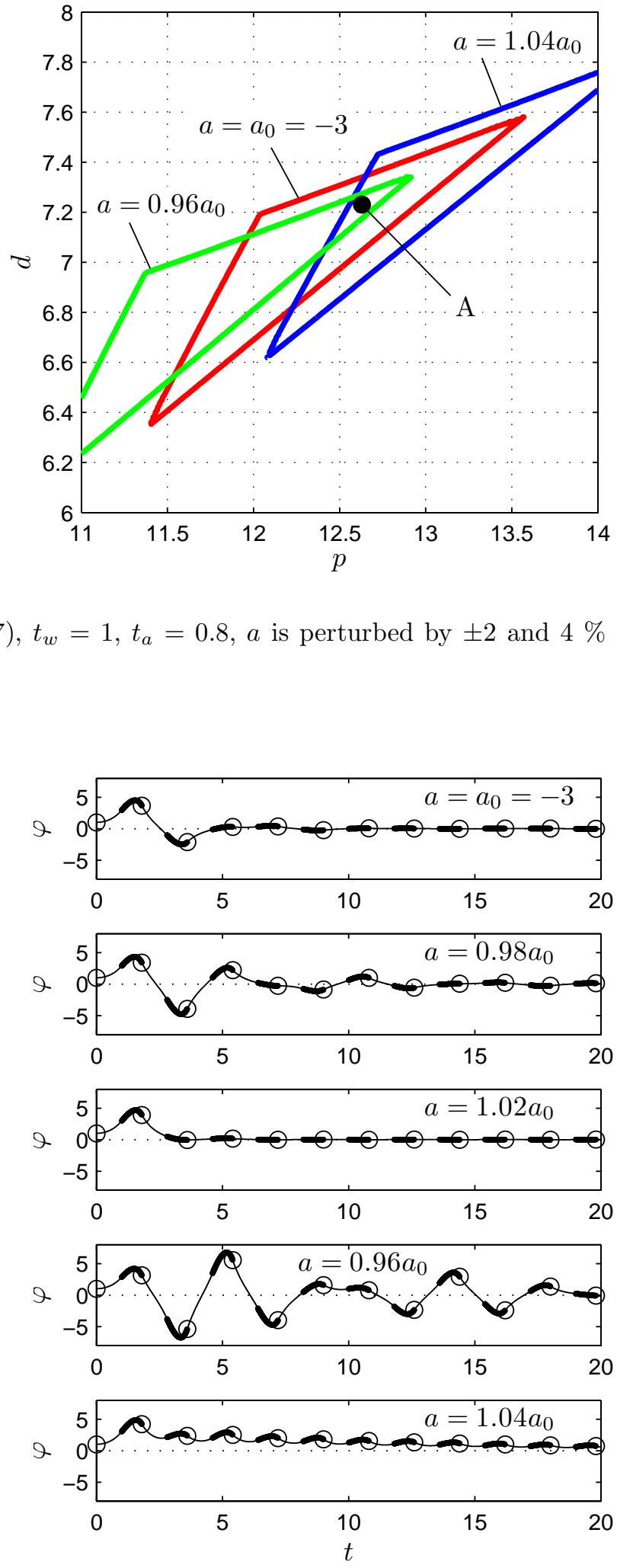

Fig. 3. Time histories for system (16) with (17), $t_{w}=1$, $t_{a}=0.8, a$ is perturbed by \pm 2 and $4 \%$ around $a_{0}=-3$. 


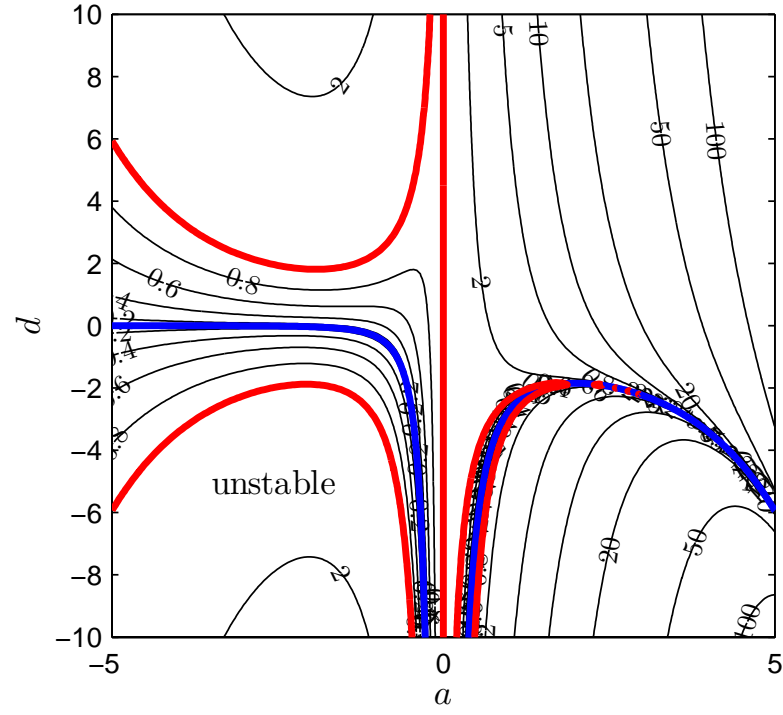

Fig. 4. Stability chart for system (23) with (17), $t_{w}=1$, $t_{a}=1$. Stability boundaries are denoted by red, deadbeat parameters are denoted by blue lines.

If the act-and-wait controller is applied in the form

$$
\dot{x}(t)=a x(t)+g(t) d \ddot{x}(t-1)
$$

with $g(t)$ being defined in (17), then the stability properties changes radically. Consider the case when $t_{w} \geq \tau$ and $t_{a} \leq \tau=1$. In this case, the monodromy matrix (that is a scalar in this case) can be constructed as

$$
\begin{aligned}
\Phi(T)=\mathrm{e}^{a T}+\int_{t_{w}}^{T} \mathrm{e}^{a(T-s)} a^{2} d \mathrm{e}^{a(s-\tau)} \mathrm{d} s & \\
& =e^{a T}\left(1+a^{2} d t_{a} \mathrm{e}^{-a \tau}\right) .
\end{aligned}
$$

The system is asymptotically stable if $|\Phi(t)|<1$. The stability diagram in the plane of $(a, d)$ is presented in Fig. 4. The stability boundaries are denoted by thick red lines, and contour lines of the decay ratio are also presented. It can be seen that the system can be stabilized for any values of $a$, although, for large positive values (or, say, for unstable open loop system), the stability regions are quite narrow. Based on equation (24), it can be seen that $\Phi(T)=0$ if

$$
d=-\mathrm{e}^{a \tau} /\left(a^{2} t_{a}\right)
$$

For this parameter combination, all the poles of the system are zero that corresponds to deadbeat control. These parameters are denoted by blue lines in Fig. 4 .

\section{CONCLUSIONS}

The stabilizing effect of the act-and-wait control concept was demonstrated in two case studies. First, a second order system subjected a PD controller was considered with a feedback delay relatively large compared to the characteristic time constant of the system. The system parameters are chosen such that the system is always unstable for any constant control gains, if the feedback is continuously applied. It was shown that this unstable system can be stabilized if the control gains are periodically switched on and off according to the act-and-wait concept.

The second system under investigation is a first order system, where the second derivative of the state is fed back. The resulting system is in fact a differential equation of advanced type that are always unstable for any parameter combinations. In this case, again, periodic switching of the control gain was found to be beneficial regarding the stability of the system. It was shown that the system can be stabilized, and furthermore, deadbeat control can be attained for some specific parameter combinations.

\section{REFERENCES}

Allwright, J.C., Astolfi, A., and Wong, H. P. (2005). A note on asymptotic stabilization of linear systems by periodic, piece-wise constant output feedback. Automatica, 41(2):339-344.

Atay, F.M. (1999). Balancing the inverted pendulum using position feedback. Applied Mathematics Letters, $12(5): 51-56$.

Boikov, I.V. (2005). The Brockett stabilization problem. Automation and Remote Control, 66(5):746-751.

Brockett, R.W. (1998). A stabilization problem. In: Open Problems in V.D. Blondel, E.D. Sontag, M. Vidyasagar, and J.C. Willems editors, Mathematical Systems and Control Theory, Communications and Control Engineering, Springer, Berlin, pp. 75-78 (Chapter 16).

Èl'sgol'c, L.'̀. (1964). Qualitative Methods in Mathematical Analysis. American MAthematical Society, Providence.

Insperger, T. (2006). Act and wait concept for timecontinuous control systems with feedback delay. IEEE Transactions on Control Systems Technology, 14(5):974-977.

Insperger T. and Stépán, G. (2007). Act-and-wait control concept for discrete-time systems with feedback delay. IEE Proceedings - Control Theory \& Applications, 1(3):553-557.

Leonov, G.A. (2002). Brockett's problem in the theory of stability of linear differential equations. St. Petersburg Math. Journal, 13(4):613-628.

Michiels, W., Niculescu, S.-I. and Moreau, L. (2004). Using delays and time-varying gains to improve the static output feedback stabilizability of linear systems: a comparision. IMA Journal of Mathematical Control and Information, 21(4):393-418.

Michiels, W. and Roose, D. (2003). An eigenvalue based approach for the robust stabilization of linear time-delay systems. International Journal of Control, $76(7): 678-686$.

Moreau, L. and Aeyels, D. (2004). Periodic output feedback stabilization of single-input single-output continuous-time systems with odd relative degree. Systems $\&$ Control Letters, 51:395-406.

Olgac, N. and Sipahi, R. (2002). An exact method for the stability analysis of time-delayed linear timeinvariant (LTI) systems. IEEE Transactions on Automatic Control, 47(5):793-977.

Stépán, G. (1989). Retarded Dynamical Systems. Harlow, Longman. 